\title{
Estudio sobre la Percepción de los Factores Involucrados en la Estimación de Precios de Viviendas: El Caso de Cajeme
}

\author{
Laura P. Lopez-Arredondo', Cynthia B. Perez ${ }^{2}$, Luis A. Castro' y Luis-Felipe Rodriguez ${ }^{1}$ \\ (1) Instituto Tecnológico de Sonora, Campus Náinari, Antonio Caso S/N y E. Kino, Colonia Villa ITSON. C.P. \\ 85130. Ciudad Obregón, Sonora. México. (e-mail: laurapaolalpz@hotmail.com; luis.castro@acm.org; \\ luis.rodriguez@itson.edu.mx) \\ (2) Instituto Tecnológico de Sonora, Unidad Guaymas, Carretera al Aeropuerto Km 3, C.P. 85400. Apartado \\ Postal 870. Guaymas, Sonora. México. (e-mail: cynthia.perez@itson.edu.mx)
}

Recibido May. 18, 2018; Aceptado Jul. 24, 2018; Versión final Sep. 13, 2018, Publicado Abr. 2019

\begin{abstract}
Resumen
El objetivo de esta investigación es obtener la información necesaria para identificar la percepción sobre los factores de mayor y menor impacto durante el proceso de valuación de viviendas. Para ello, realizaron diez entrevistas semiestructuradas, las cuales se analizaron mediante Teoría Fundamentada. Los resultados de este trabajo sugieren que tanto los compradores como los vendedores de viviendas perciben a la ubicación, las proporciones y las condiciones de las viviendas como factores determinantes para estimar el valor de éstas. Los resultados de este trabajo pueden ser utilizados para mejorar el diseño de modelos predictivos para la estimación de precios de bienes raíces utilizados en plataformas en línea dedicadas a la compra-venta de bienes raíces. Así mismo, el estudio proporciona las bases necesarias para establecer las variables o parámetros que mejor se adapten al algoritmo predictivo para la estimación de precios de viviendas en la región sur del estado de Sonora, México.
\end{abstract}

\section{Study about the Perception of Factors Involved in Housing Price Estimation: The Case of Cajeme}

\begin{abstract}
This work aims at obtaining the necessary information for identifying the perceived factors of higher or lower impact during the housing valuation process. Ten semi-structured interviews were conducted, and analyzed using Grounded Theory. The results of this work suggest that both buyers and sellers of households perceive the location, proportions and housing conditions as determining factors to estimate the value of these properties. The results of this work can be used to improve the design of predictive models for real estate which are used in online platforms. Furthermore, the study provides the bases to establish the variables or parameters used in the predictive algorithm for estimating housing prices in the southern region of the state of Sonora, Mexico.
\end{abstract}

Keywords: qualitative research; grounded theory; determining factors; housing valuation; real estate market; real estate buyers 
Estudio sobre la Percepción de los Factores Involucrados en la Estimación de Precios Lopez-Arredondo

\section{INTRODUCCIÓN}

Para muchas personas, comprar o vender una casa es una de las decisiones más importantes en su vida e involucra un amplio umbral de incertidumbre acerca del valor a asignarle a la vivienda, puesto que, además de la asequibilidad del inmueble, otros factores como la ubicación o el panorama de la inversión a largo plazo afectan en mayor o menor medida al proceso de valuación. Debido a esto, la información relacionada con el valor de las viviendas es fundamental para los investigadores, organismos de gobierno, empresas de bienes raíces, vendedores de viviendas y para los propietarios de inmuebles. Esquer (2012) menciona que los métodos de valuación de inmuebles suelen ser bastante lentos y tediosos debido a que requieren de una investigación bien fundada y, en general, los procedimientos actuales deben basarse en información confiable para obtener resultados más precisos.

Asimismo, el impacto de los factores durante el proceso de valuación de viviendas varía dependiendo de la percepción que se tenga sobre estos. Carterette y Friedman (1982) definen la percepción como una parte primordial de la conciencia que consta de hechos abstraídos del mundo externo y, por tanto, se refiere a la realidad tal como es experimentada. Según Arias (2006), "la percepción depende de la actividad de receptores que son afectados por procesos provenientes del mundo físico y puede entonces definirse como el resultado del procesamiento de información que consta de estimulaciones a receptores en condiciones que en cada caso se deben parcialmente a la propia actividad del sujeto". Con base en lo anterior, se puede decir que, ya sea que se desee vender o comprar una vivienda o inmueble, tener noción de cómo se determina su costo brinda control sobre el patrimonio en cuestión; además, proporciona seguridad en caso de que se necesite realizar una transacción con dicho inmueble.

Bell et al. (2015) mencionan que en el ámbito inmobiliario hay muchos métodos prescritos para obtener datos de mercado y otra información crítica; dichos métodos pueden ser de carácter cualitativo o cuantitativo. Los métodos más utilizados para este contexto son los cuantitativos debido a que, en muchos casos, los problemas inmobiliarios se prestan de manera predecible a datos cuantificables. Sin embargo, como ya se mencionó, los participantes del mercado inmobiliario tienen percepciones propias que pueden influir en los precios de venta y otros datos cuantificables. Debido a esto, el autor argumenta que el uso de métodos cualitativos puede proporcionar una mejor comprensión de los problemas y las motivaciones dentro del mercado. Durante la revisión de la literatura, se encontró que existe una considerable cantidad de investigadores interesados en el análisis cualitativo como método para obtener la percepción de las personas respecto a diversas cuestiones en áreas como la medicina, psicología, tecnología, gestión empresarial, estudio de mercados, entre otras (Figueira et al 2009; Bedoya et al., 2017; Festen et al. 2014; Riley et al. 2009; Bravo et al., 2018; Oliveira et al. 2016; Padilla et al., 2017; Pérez-Benedito et al., 2017; Kamagahara et al., 2016). Sin embargo, hay escasez de estudios en donde se lleve a cabo el análisis cualitativo para el contexto de bienes raíces.

En el ámbito inmobiliario, Kibet et al. (2016) llevó a cabo un estudio para evaluar el efecto de los factores socioculturales en las inversiones inmobiliarias. Ellos aplicaron una encuesta a una muestra de 300 propietarios de propiedades inmobiliarias comerciales en la ciudad de Kisumu en Kenia. Los datos fueron analizados tanto cualitativa como cuantitativamente y los resultados revelaron que los factores socioculturales influyen en gran medida en la inversión inmobiliaria. De igual forma, Kakulu (2014) describe una serie de estrategias para el análisis cualitativo y diversos métodos para el análisis de datos en el campo de bienes raíces en Nigeria. Asimismo, el autor argumenta que las futuras investigaciones de bienes raíces en dicho país deben tomar en cuenta las percepciones de las personas puesto que es un área que aún está poco investigada en dicha región. El autor recomienda ampliamente el uso de estrategias de investigación de fenomenología y aplicación de casos de estudio, métodos de recolección de datos de grupos focales y, el uso de una herramienta innovadora de análisis de contenido para el análisis de datos y la interpretación de los hallazgos, de tal manera que remarcan la importancia de este tipo de análisis. A pesar de ello, son muy pocos los estudios que se realizan bajo el contexto inmobiliario y sobre todo que utilicen métodos cualitativos de investigación.

Este trabajo tiene como objetivo identificar la percepción sobre los factores de mayor y menor impacto tomados en cuenta durante el proceso de valuación de una vivienda por parte de los compradores y vendedores de viviendas que residen en el municipio de Cajeme, ubicado en la región sur del estado de Sonora. El enfoque de teoría fundamentada, se basa en recopilar y analizar datos obtenidos mediante entrevistas semi-estructuradas. Una vez que se identificaron diversos patrones, categorías y similitudes en los datos analizados, los resultados demostraron que tanto los compradores como los vendedores de viviendas perciben a la ubicación, las proporciones y las condiciones de las viviendas como factores determinantes para estimar el valor de estas. Lo anterior proporciona beneficios para las personas de la región involucradas en la compra-venta de inmuebles, puesto que permitirá hacer aún más robusto el método de valuación a emplear al considerar el impacto que tiene cada uno de los factores. De igual manera, proporcionará las bases necesarias para establecer los parámetros de un algoritmo predictivo para la estimación del valor de las viviendas de la región. 


\section{FENÓMENO BAJO ESTUDIO}

Según datos del Instituto Nacional de Estadística y Geografía (INEGI, 2010), la población total del municipio de Cajeme fue de 409,310 habitantes en 2010, de los cuales más del 72\% se concentraban en Ciudad Obregón, siendo ésta la cabecera municipal donde también se encontraba la mayor actividad económica. Así mismo, se calculó una tasa de crecimiento poblacional del 1.72\% para el periodo 2011-2015 con base en históricos de 2005-2010. La agricultura es la principal base para el desarrollo económico del municipio, sin embargo, a través del tiempo se han introducido nuevas tecnologías y presentado nuevos productos al mercado (IMIP, 2017). La estructura urbana de la cabecera municipal se caracteriza por concentrar los edificios y monumentos más antiguos en el centro y por mantener gran parte de las viviendas de los habitantes en la periferia, propiciando el crecimiento urbano en la zona norte y sureste de la ciudad (Ayuntamiento de Cajeme, 2011). En cuanto al tema de seguridad pública, Cajeme fue el segundo municipio con mayor número de delitos registrados en 2012, lo cual representó un aumento gradual en la inseguridad con respecto en años anteriores (INEGI, 2012). Respecto al ámbito inmobiliario, en 2015 había un total de 128,490 viviendas particulares en el municipio, de las cuales más del $98 \%$ contaban con servicios básicos de energía eléctrica, agua de la red pública y drenaje. Además, el número de habitantes promedio por vivienda fue de 3.4 personas (INEGI, 2015). En el mismo año, se proyectó un notable crecimiento en el mercado inmobiliario del municipio, puesto que doce nuevos fraccionamientos se encontraban en proceso de desarrollo (CANADEVI, 2015).

En los últimos años, los problemas relacionados con el análisis del mercado inmobiliario han crecido muy rápido ya que este conocimiento es muy relevante para las predicciones inmobiliarias, las inversiones y las cuestiones relacionadas con los impuestos (Del Giudice et al. 2017). Debido a ello, la valuación de viviendas es un tema que ha sido constantemente explorado e investigado. Sin embargo, el impacto de los factores clave para determinar el valor de los inmuebles varía dependiendo de los aspectos socioculturales y de la percepción sobre el tema de valuación de viviendas que se tenga en cada región. Dicho impacto se puede ver afectado por el tipo de personas que viven en alguna comunidad, las condiciones de las viviendas de dicha zona, entre otros. Realizar una investigación de carácter cualitativo permite analizar la percepción que tienen los compradores y vendedores de cierta región sobre los factores clave para estimar el valor de las viviendas; a través de este estudio, se pretende identificar cuáles son los más relevantes en el municipio de Cajeme, Sonora. En la Tabla 1 se muestra una descripción de los factores estándar a tomar en cuenta al momento de realizar la estimación del valor de alguna vivienda. Dichos factores serán contemplados durante la realización de este trabajo de investigación.

Tabla 1: Factores para la valuación de viviendas.

\begin{tabular}{|c|c|c|}
\hline Factor & Concepto & Referencias \\
\hline Ubicación & $\begin{array}{l}\text { Es uno de los factores con mayor influencia en el valor de las } \\
\text { viviendas. El precio de venta o el costo del metro cuadrado de una } \\
\text { vivienda varían dependiendo de la región en donde se encuentre. } \\
\text { Esto, debido a la demanda que tiene la zona, las condiciones del } \\
\text { vecindario, el grado de desarrollo a su alrededor, entre otros. }\end{array}$ & $\begin{array}{l}\text { Basu et al. (1998); } \\
\text { Bourassa et al. (2007). }\end{array}$ \\
\hline Tipo de propiedad & $\begin{array}{l}\text { El valor de la vivienda se puede ver afectado por el tipo de } \\
\text { propiedad, es decir, si el inmueble se trata de una casa o un } \\
\text { departamento. Aquí también se ven involucradas la cantidad de } \\
\text { habitaciones y tipos de habitaciones con las que cuenta la } \\
\text { propiedad. }\end{array}$ & $\begin{array}{l}\text { Daniels et al. (1999); } \\
\text { Zietz et al. (2008). }\end{array}$ \\
\hline $\begin{array}{l}\text { Condiciones de } \\
\text { vivienda }\end{array}$ & $\begin{array}{l}\text { En este punto se tocan aspectos como: el año de construcción, } \\
\text { condiciones del techo, condiciones del piso, condiciones de las } \\
\text { paredes, material exterior, tipo de tejado, reparaciones, vistas e } \\
\text { iluminación. }\end{array}$ & $\begin{array}{l}\text { Haurin et al. (1996); } \\
\text { Zietz et al. (2008); } \\
\text { Bourassa et al. (2007). }\end{array}$ \\
\hline $\begin{array}{l}\text { Proporciones de la } \\
\text { vivienda }\end{array}$ & $\begin{array}{l}\text { Involucra diversos aspectos de la vivienda, tales como: área, } \\
\text { perímetro, número de pisos, cochera, estacionamiento y patio. }\end{array}$ & $\begin{array}{l}\text { Daniels et al. (1999); } \\
\text { Haurin et al. (1996); } \\
\text { Zietz et al. (2008). }\end{array}$ \\
\hline Instalaciones & $\begin{array}{l}\text { La antigüedad de la vivienda se relaciona directamente con las } \\
\text { condiciones de sus instalaciones debido al deterioro que pueden } \\
\text { presentar. Los tipos de instalaciones van desde instalaciones } \\
\text { eléctricas, de agua potable, red de saneamiento, de ventilación o } \\
\text { servicios. }\end{array}$ & $\begin{array}{l}\text { Zietz et al. (2008); } \\
\text { Schram (2006). }\end{array}$ \\
\hline Sitios cercanos & $\begin{array}{l}\text { Los sitios cercanos al inmueble pueden tener influencias positivas o } \\
\text { negativas en los precios de las viviendas. Entre los ejemplos de } \\
\text { sitios cercanos se incluyen: escuelas, supermercados, hospitales o } \\
\text { transporte público. }\end{array}$ & $\begin{array}{l}\text { Basu et al. (1998); Zietz } \\
\text { et al. (2008); Bourassa } \\
\text { et al. (2007). }\end{array}$ \\
\hline
\end{tabular}




\section{METODOLOGÍA}

El propósito de esta sección es describir el método de la teoría fundamentada que se utilizó para llevar a cabo el trabajo de investigación. A continuación, se presenta un listado de los pasos realizados durante la investigación con la finalidad de lograr los objetivos planteados. Posteriormente, se describen las preguntas de investigación, el proceso de recopilación de datos y el análisis de los mismos. Como se mencionó anteriormente, para llevar a cabo esta investigación se utilizó un enfoque de teoría fundamentada. Strauss y Corbin (1994) definen a la teoría fundamentada como una metodología generalizada para desarrollar teoría sobre un fenómeno de estudio en particular, basándose en datos recolectados y analizados sistemáticamente. La teoría evoluciona durante el proceso de investigación, y lo hace a través de la interacción continua entre el análisis y la recopilación de datos. Glaser y Strauss (2017) argumentan que la teoría fundamentada exige identificar categorías teóricas que son derivadas de los datos mediante la utilización de un método comparativo e iterativo. Es decir, se compara el contenido de diversas sesiones de entrevistas o de observación con los conceptos teóricos nacientes del esfuerzo por identificar los temas fundamentales (Wells et al. 1995; Barnes, 1996).

\section{Proceso de investigación}

El proceso de investigación que se llevó a cabo, se basó en una serie de pasos definidos por Glaser y Strauss (2017), tal como se muestra en la Figura 1.

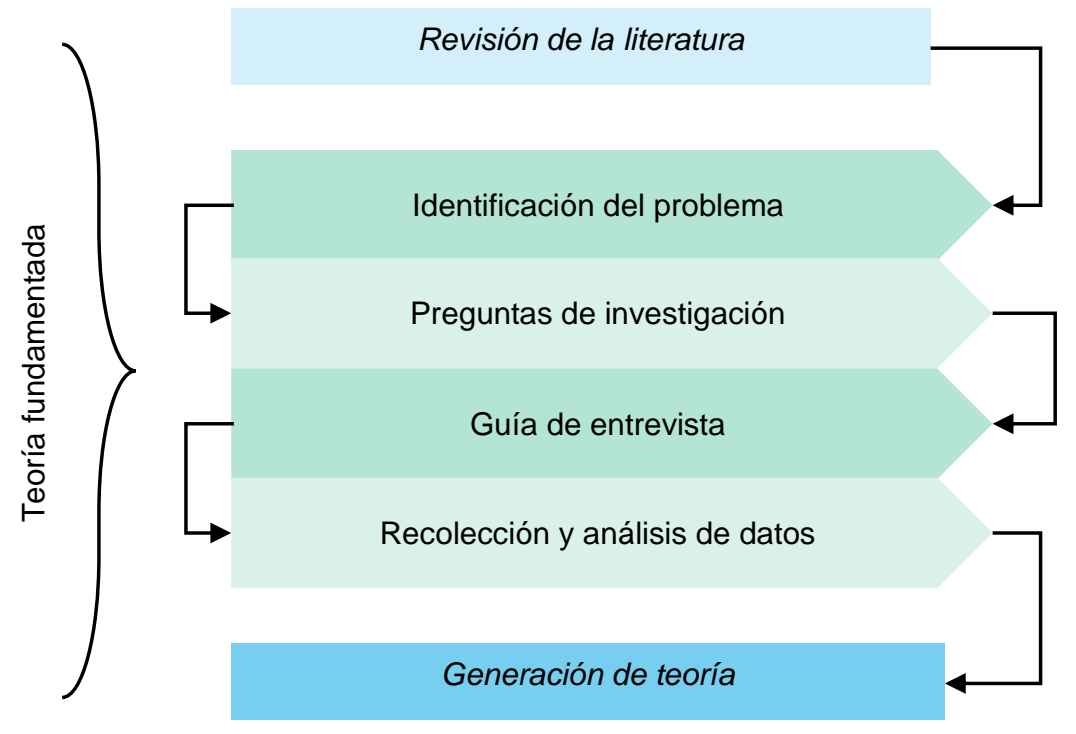

Fig. 1: Metodología de teoría fundamentada como generadora de teoría a partir de los datos.

\section{Preguntas de investigación}

Agee (2009) menciona que muchos investigadores con enfoque en los estudios cualitativos ven una simple pregunta como un punto de partida para su investigación. Una vez que la pregunta se encuentra situada satisfactoriamente en su lugar, el estudio puede comenzar. Posterior a la detección del problema o fenómeno de estudio, se identificaron tres preguntas de investigación. En este caso, las preguntas de investigación buscan explorar todos aquellos factores que toman en cuenta los individuos al momento de estimar el valor de una vivienda, así como todos aquellos que ignoran. Se inicia planteando una pregunta general que se complementa con otras dos cuestiones específicas: 1) ¿Cuál es la percepción del individuo sobre los factores que determinan el valor de una vivienda en la región sur del estado de Sonora?; 2) ¿Cuáles son los factores de mayor y menor impacto durante la estimación de costo de una vivienda por parte de los compradores?; 3) ¿Cuáles son los factores de mayor y menor impacto durante la estimación del precio de venta por parte de los vendedores?

\section{Participantes}

Con el objetivo de determinar un adecuado tamaño muestral para este trabajo de investigación, se empleó el método del muestreo selectivo propio de la investigación cualitativa. Martínez-Salgado (2012) menciona que, a diferencia del muestreo probabilístico, "la indagación cualitativa, en cambio, elige las unidades de estudio con un propósito: lograr un conocimiento intensivo, profundo y detallado de y sobre los casos en los que tiene lugar el fenómeno de interés, generalizable para otras situaciones en las que dicho fenómeno ocurre". Además 
del propósito anterior, es importante tomar en cuenta otros factores al momento de delimitar el tamaño de la muestra; tal es el caso de las semanas disponibles para realizar la investigación, ya que el análisis de los datos cualitativos recolectados es bastante riguroso y toma mucho tiempo al ser llevado a cabo de forma manual (Basit, 2003). En este caso, se contaba solamente con 16 semanas para realizar el estudio. Por ello, se optó por establecer un número limitado de sujetos, los suficientes para representar al sector clave de compradores y vendedores de viviendas en la región estudiada.

Se decidió aplicar un total de 10 entrevistas a habitantes del municipio de Cajeme, ubicado en la región sur del estado de Sonora que compartían rasgos y estilos de vida predominantes en la región. De ellos, 5 correspondían a personas que cuentan con experiencia en cuanto a la compra de inmuebles o personas con planes de adquirir una vivienda. Los otros 5 participantes correspondían a personas con experiencia en la venta de inmuebles; dicha experiencia podía haber sido obtenida con apoyo por parte de profesionales de bienes raíces o por la venta directa de algún inmueble. Las entrevistas tuvieron una duración de 30 a 50 minutos, de las cuales se tuvieron que descartar 2 por falta de claridad y consistencia en la información obtenida (E5 y E6). Se identificó que las 8 entrevistas restantes compartían bastante información en común, lo que hacía posible acotar aún más el número de entrevistas a analizar. Finalmente se eligieron 4 para llevar a cabo el análisis cualitativo. La decisión se basó en la calidad de la información proporcionada, en la similitud de las ideas expuestas y en el rango de edad de los participantes, es decir, personas de 30 años o más. Para esto, los participantes de las entrevistas seleccionadas para el análisis fueron E1, E4, E7 y E9. Dos de las entrevistas son de personas con experiencia en la compra de viviendas y las otras dos entrevistas son de personas con experiencia en la venta de viviendas. Específicamente, el sujeto E1 realizó la compra de una vivienda unifamiliar aislada en un vecindario con nivel socioeconómico medio; el sujeto E4 llevó a cabo la venta de una vivienda unifamiliar aislada en un vecindario con nivel socioeconómico bajo; E7 adquirió una vivienda unifamiliar aislada en un vecindario con nivel socioeconómico alto y el sujeto E9 vendió una vivienda unifamiliar adosada en un vecindario con nivel socioeconómico medio. En la Tabla 2 se puede apreciar el perfil general de los participantes que fueron entrevistados.

Tabla 2: Perfil de los participantes.

\begin{tabular}{|c|c|c|c|c|c|c|}
\hline Participante & Edad & $\begin{array}{c}\text { Plan de } \\
\text { compra }\end{array}$ & $\begin{array}{c}\text { Experiencia de } \\
\text { compra }\end{array}$ & $\begin{array}{c}\text { Presencia durante } \\
\text { el proceso de } \\
\text { venta }\end{array}$ & $\begin{array}{c}\text { Experiencia de } \\
\text { venta }\end{array}$ & $\begin{array}{c}\text { Calidad en la } \\
\text { información }\end{array}$ \\
\hline E1 & 44 & & $\mathrm{X}$ & & & $\mathrm{X}$ \\
\hline E2 & 36 & $\mathrm{X}$ & & & & $\mathrm{X}$ \\
\hline E3 & 25 & $\mathrm{X}$ & & & & $\mathrm{X}$ \\
\hline E4 & 60 & & & & & $\mathrm{X}$ \\
\hline E5 & 38 & & $\mathrm{X}$ & & $\mathrm{X}$ & \\
\hline E6 & 37 & & & & & $\mathrm{X}$ \\
\hline E7 & 34 & & $\mathrm{X}$ & & $\mathrm{X}$ & \\
\hline E8 & 46 & & & & $\mathrm{X}$ & $\mathrm{X}$ \\
\hline E9 & 30 & & & & & \\
\hline E10 & 45 & & & & & $\mathrm{X}$ \\
\hline
\end{tabular}

\section{Recolección de datos}

Para llevar a cabo la recolección de los datos se diseñó una guía de entrevista de tipo semiestructurada, tomando como base las preguntas de investigación mencionadas anteriormente. Cohen et al. (2006) menciona que las guías de entrevistas semiestructuradas proporcionan un conjunto claro de instrucciones para los entrevistadores y, puede generar datos cualitativos fiables y comparables. También brindan la oportunidad de identificar nuevas formas de ver y comprender el tema bajo estudio. Por otro lado, Clifford et al. (2016) menciona que las entrevistas semiestructuradas se desarrollan de una manera conversacional, ofreciendo a los participantes la oportunidad de explorar cuestiones que ellos crean relevantes. Este tipo de entrevista semiestructurada tiene como objetivo alentar al sujeto entrevistado para que narre algún evento importante de su vida y del contexto social (Muylaert et al. 2014). La guía o protocolo de entrevista llevada a cabo en este estudio, cubre los siguientes temas: i) Situación del individuo: Preguntas generales sobre antecedentes del participante, incluyendo su situación actual personal y de la vivienda, así como los conocimientos base que posee sobre la valuación de viviendas; ii) Experiencias con la compra o venta de viviendas: El propósito de estas preguntas es conocer cómo fue el proceso de compra o venta de la vivienda en cuestión; iii) Percepción de factores clave: Se realizan preguntas para conocer la percepción que tiene el participante sobre los diversos factores involucrados en la estimación del valor de las viviendas. 


\section{Análisis de los datos}

Una vez concluidas las entrevistas, se realizó su transcripción para proceder con el análisis de línea por línea. El objetivo de llevar a cabo el análisis de las trascripciones es profundizar aún más en las historias compartidas por los participantes, en busca de información relevante que responda a las preguntas de investigación planteadas. Por lo tanto, es necesario analizar e interpretar los datos para construir un sentido e ir generando la teoría a partir de estos. El proceso de análisis se puede ver reflejado en la Figura 2, en donde la codificación abierta se refiere a descomponer la información línea por línea, la codificación axial sugiere la creación de categorías, en la codificación selectiva se elige una categoría base y, por último, se procede con la generación de una teoría.

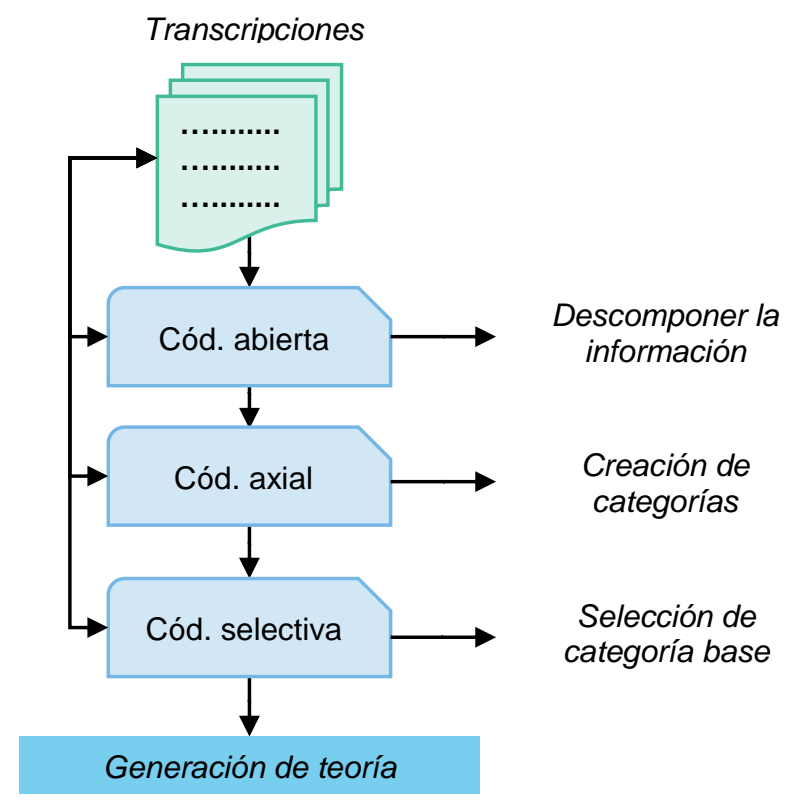

Fig. 2: Tipos de codificación para el análisis de datos.

\section{Codificación abierta}

Codificar los datos significa identificar todas y cada una de las ideas representadas en cada línea de texto de las transcripciones. Para esto, se debe dar un nombre clave a cada nueva idea, preferentemente que sean palabras claras y concisas (Grams, 2001). La codificación abierta incluye conceptos de etiquetado, definiendo y desarrollando categorías basadas en sus propiedades y dimensiones. Durante este proceso, los conceptos surgen de los datos brutos y luego se agrupan en categorías conceptuales. El objetivo es construir un marco preliminar descriptivo y multidimensional para su análisis posterior. El proceso por sí solo asegura la validez del trabajo debido a que se construye directamente a partir de los datos brutos (Khandkar, 2009). Durante esta fase, se fraccionaron los datos de las trascripciones para obtener diversos códigos que después se agruparían por categorías. En la Figura 3 se puede observar un fragmento de la transcripción de una entrevista y su respectiva codificación abierta, las palabras en rojo corresponden a las etiquetas resultantes según se analizaba el texto línea por línea.

\section{Codificación axial}

Strauss y Corbin (2002) definen la codificación axial como "el proceso de relacionar las categorías a sus subcategorías, denominado 'axial' porque la codificación ocurre alrededor del eje de una categoría, y enlaza las categorías en cuanto a sus propiedades y dimensiones". Es por ello que el propósito de la codificación axial consiste en iniciar con la reagrupación de los datos que se fracturaron durante la codificación abierta. En la codificación axial, las categorías se relacionan con sus subcategorías para formar unas explicaciones más precisas y completas sobre los fenómenos (Strauss et al. 1998). En esta fase, se analizaron los códigos resultantes de la codificación abierta y se agruparon por categorías, subcategorías, propiedades y dimensiones, lo cual resultó en una mejor comprensión del escenario bajo estudio. En la Tabla 3 se muestran algunos datos resultantes del proceso de codificación axial; se encontraron tres categorías principales, doce subcategorías, sesenta propiedades y ciento veinte dimensiones. 
Fig. 3: Fragmento de codificación abierta realizada a transcripción de E4.

\begin{tabular}{|ccc|}
\hline $\begin{array}{c}6 \\
7\end{array}$ & $\mathrm{~L} 4$ & ¿Y validó de alguna manera el precio de venta? \\
8 & & tema lo validé, por el tipo de casa que tenía, más o menos le pregunté a alguien que sabía del \\
9 & & valuación vivienda], me dijo "pues por lo que es, por la zona, en dónde está y la construcción \\
10 & pues vale más o menos tanto, [Factores determinantes] puedes variar un poco más o poco \\
11 & menos ese precio, pero eso es aproximadamente". [Estimación de precio de venta] \\
12 & Entonces yo tomé como referencia ese precio que me dio esa persona que sabe sobre \\
13 & valuación de viviendas y modifiqué un poco el precio [Ajuste de precio de venta] ya viendo \\
14 & yanalizando bien la casa [Verificar condiciones de vivienda] y las ofertas de los compradores \\
15 & que tenía en ese momento. [Analizar ofertas]
\end{tabular}

Las categorías principales se definen a continuación: i) Contexto actual del individuo: Concentra toda aquella información en torno a la situación actual del individuo bajo estudio. Es decir, involucra aspectos de su vida personal, características esenciales de su vivienda (ej. si es propia o rentada), así como su nivel de conocimientos sobre el tema de valuación de viviendas; ii) Experiencias del individuo: Se refiere a todos los aspectos relacionados con la experiencia, ya sea en la compra de viviendas o con la venta de estas. Así mismo, involucra propiedades como el tipo de experiencia, las ventajas, los problemas ocurridos, así como las habilidades y conocimientos puestos en marcha durante el proceso de compra o de venta; iii) Percepción de factores estimación: Aquí se concentra información importante sobre la percepción del individuo en cuanto a los diversos factores involucrados durante la estimación del valor de una vivienda, ya sea para su compra o venta, tales factores son: ubicación, sitios cercanos, vecinos, proporciones de la vivienda, condiciones de la vivienda, instalaciones, seguridad, terreno excedente de la vivienda. Así mismo, se determina el grado de importancia (alto o bajo) que percibe el individuo bajo estudio para cada uno de dichos factores, las ventajas y desventajas percibidas y demás información relevante que permite profundizar en la percepción que tienen de los mismos.

Tabla 3: Fragmento de codificación axial realizada durante análisis de datos.

\begin{tabular}{|c|c|c|c|}
\hline Categorías & Subcategorías & Propiedades & Dimensiones \\
\hline $\begin{array}{l}\text { I. Contexto actual } \\
\text { del individuo }\end{array}$ & & $\begin{array}{l}\text { Vida personal } \\
\text { Características de la vivienda } \\
\text { Habilidades y conocimientos } \\
\text { sobre valuación }\end{array}$ & $\begin{array}{l}\text { Edad, Estado civil, .... } \\
\text { Rentada, Compartida, propia, } \\
\text { remodelada, ... } \\
\text { Alto, medio, bajo }\end{array}$ \\
\hline \multirow[t]{2}{*}{$\begin{array}{l}\text { II. Experiencias } \\
\text { del individuo }\end{array}$} & Compra de viviendas & $\begin{array}{l}\text { Tipo de experiencias } \\
\text { Ventajas } \\
\text { Problemas } \\
\text { Habilidades y conocimientos } \\
\text { Tipo plan de compra }\end{array}$ & $\begin{array}{l}\text { Satisfactoria, insatisfactoria } \\
\text { Asesoría, claridad en procesos de } \\
\text { compra, facilidades de pago, ... } \\
\text { Estimación de costos, ... } \\
\text { Largo o corto plazo }\end{array}$ \\
\hline & Venta de viviendas & $\begin{array}{l}\text { Tipo de experiencia } \\
\text { Ventajas } \\
\text { Problemas } \\
\text { Habilidades y conocimientos }\end{array}$ & $\begin{array}{l}\text { Positiva o Negativa } \\
\text { Asesoría, Uso de Tecnología, ... } \\
\text { Periodos de espera, ... } \\
\text { Intereses de compradores, ... }\end{array}$ \\
\hline \multirow[t]{2}{*}{$\begin{array}{l}\text { III. Percepción de } \\
\text { factores } \\
\text { estimación }\end{array}$} & $\begin{array}{l}\text { Factores } \\
\text { determinantes para } \\
\text { compra }\end{array}$ & Nivel de impacto & Alto, Medio, Bajo \\
\hline & $\begin{array}{l}\text { Factores } \\
\text { determinantes para } \\
\text { venta }\end{array}$ & Nivel de impacto & Alto, Medio, Bajo \\
\hline
\end{tabular}


Tabla 3 (continuación)

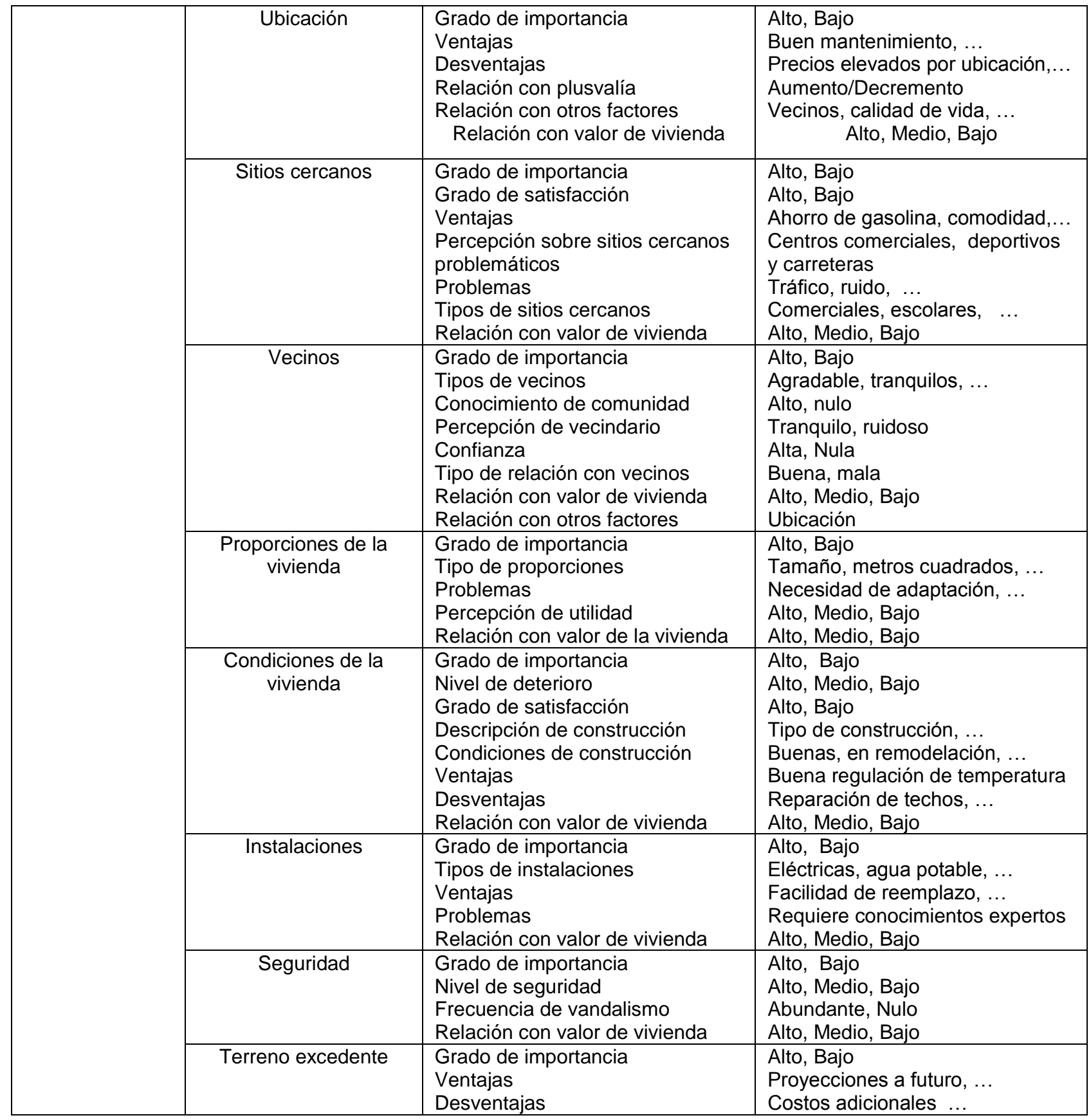

\section{RESULTADOS}

Una vez concluido el análisis mediante la codificación abierta y la codificación axial, se comenzó el proceso de integrar y refinar la teoría, también llamado codificación selectiva. Strauss y Corbin (1998) argumentan que, al igual que en todas las fases del análisis, la integración de la teoría requiere de la interacción constante entre el analista y los datos. Esta fase inicia con la selección de una categoría medular que representa el tema principal de la investigación. En este caso las preguntas de investigación no cambiaron conforme avanzaba el análisis, esto debido a que los datos continuaron relacionados con las mismas preguntas. Se eligió la tercera categoría como núcleo central para darle respuesta a las preguntas de investigación planteadas. Es decir, la categoría sobre la "percepción de los factores de estimación".

En la Figura 4 se puede observar la evolución del proceso de análisis de datos mediante las fases de codificación abierta, axial y selectiva. Se puede apreciar que los códigos resultantes se clasificaron en tres categorías principales (Contexto actual del individuo, Experiencias del individuo, Percepción de los factores) que interactuaban con algunas subcategorías (Factores determinantes en la compra/venta de viviendas, Experiencias con la compra/venta de viviendas), propiedades y dimensiones (Grado de importancia, Tipo de experiencia, Habilidades/Conocimientos, Contexto de vivienda actual, Contexto personal). Con la elección de 
la categoría central, se reordenaron las relaciones entre categorías, subcategorías, propiedades y dimensiones, quedando la categoría sobre la percepción de los factores de estimación al centro y manteniendo una relación con las experiencias de los individuos, el contexto actual del individuo y el listado de los factores determinantes para la compra/venta de viviendas.

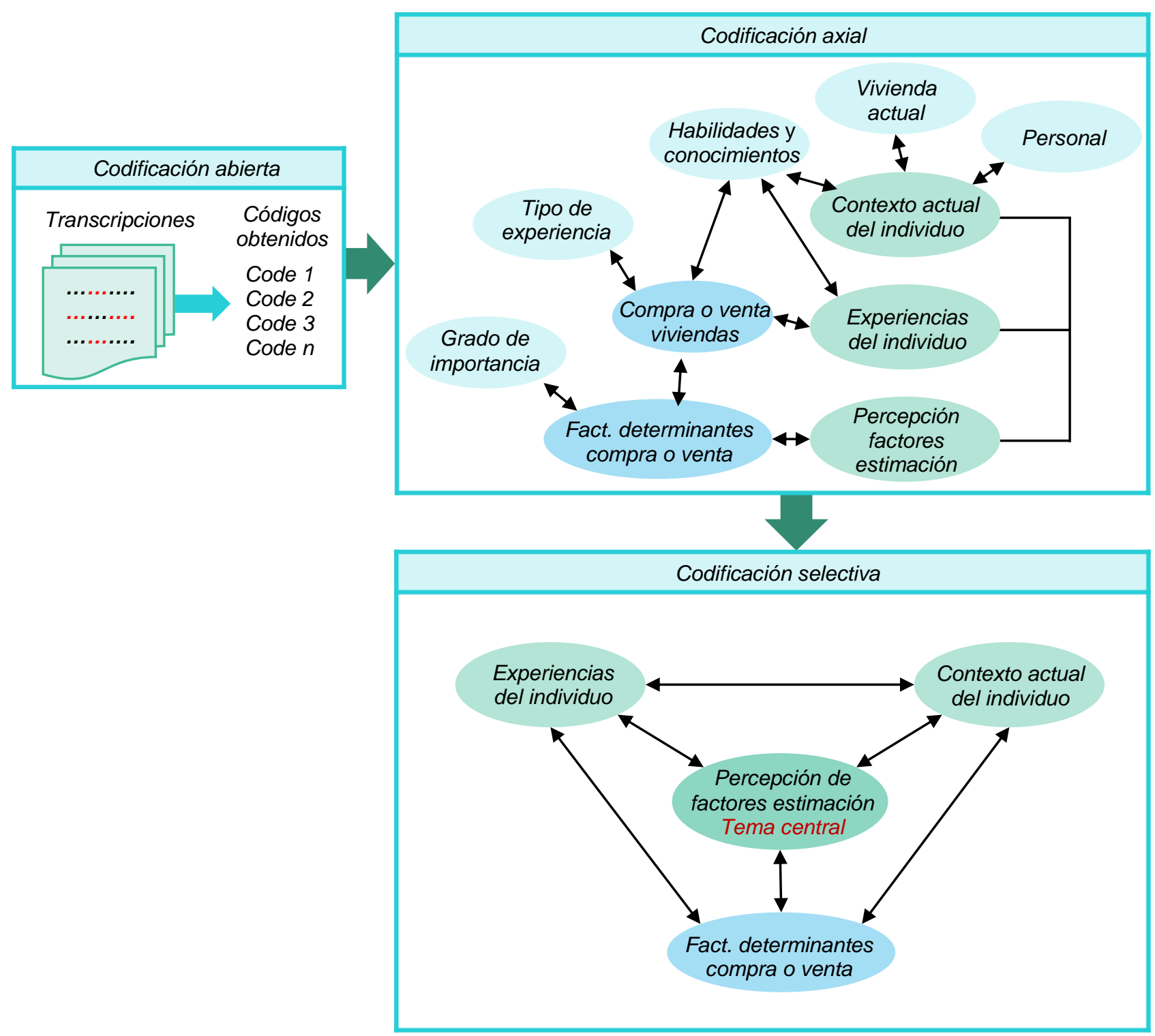

Fig. 4: Proceso de análisis de los datos: Codificación abierta, axial y selectiva.

\section{Percepción general sobre los factores que determinan el valor de una vivienda}

Tal como se menciona anteriormente, el análisis de las entrevistas arrojó a la categoría sobre la "percepción de los factores de estimación" como la central. Dicha categoría, en relación con las otras dos categorías identificadas, permite responder a las preguntas de investigación planteadas. En la Figura 5 se puede observar que los compradores y vendedores de viviendas de la región perciben tres factores como determinantes clave del valor de las viviendas, es decir: la ubicación, las proporciones y las condiciones de la vivienda. Todos los individuos entrevistados coincidieron en que dichos factores son los más importantes al momento de determinar, ya sea el costo o el precio de venta del inmueble. Por ejemplo, E7 (comprador) hizo énfasis en la relación directa que existe entre la ubicación de una vivienda y su costo: "si es en una zona de las afueras de la ciudad pues está más barata, si es céntrica ahí aumenta más el costo y si es en un residencial pues aún más cara todavía" (E7). Por otro lado, E9 (vendedor) menciona que, para los vendedores, la ubicación "es importante por la plusvalía que le puedes dar al inmueble" (E9). En cuanto a las proporciones de la vivienda, E4 (vendedor) comenta: "Considero muy importante las proporciones. Sí tienen mucho que ver para sacar el precio de la vivienda, por los metros cuadrados. Tienes que ver el factor ese, es muy importante, el tamaño del terreno. Es primordial" (E4). Así mismo, E1 (comprador) afirma que las condiciones de la vivienda "Sí, tienen mucho que ver para la valuación de una vivienda, el tipo de material y construcción que 
tenga es uno de los factores más importantes en los que todas las personas y valuadores se fijan o toman en cuenta" (E1). Sin embargo, las opiniones de los participantes difieren en un par de factores, los compradores consideran determinantes para el costo de la vivienda a la seguridad y el terreno excedente. E1 (comprador) asegura que "por el riesgo que hay en el vecindario debería valer menos la casa" (E1). Es decir, si el nivel de seguridad en la zona es bajo, el costo de la vivienda debería disminuir por dicho factor. Por otro lado, E7 (comprador) comenta porqué piensa que el terreno excedente determina el costo: "La casa que elegí no se me hizo cara, tuve que dar un extra de dinero pero fue porque yo compré terreno excedente" (E7).

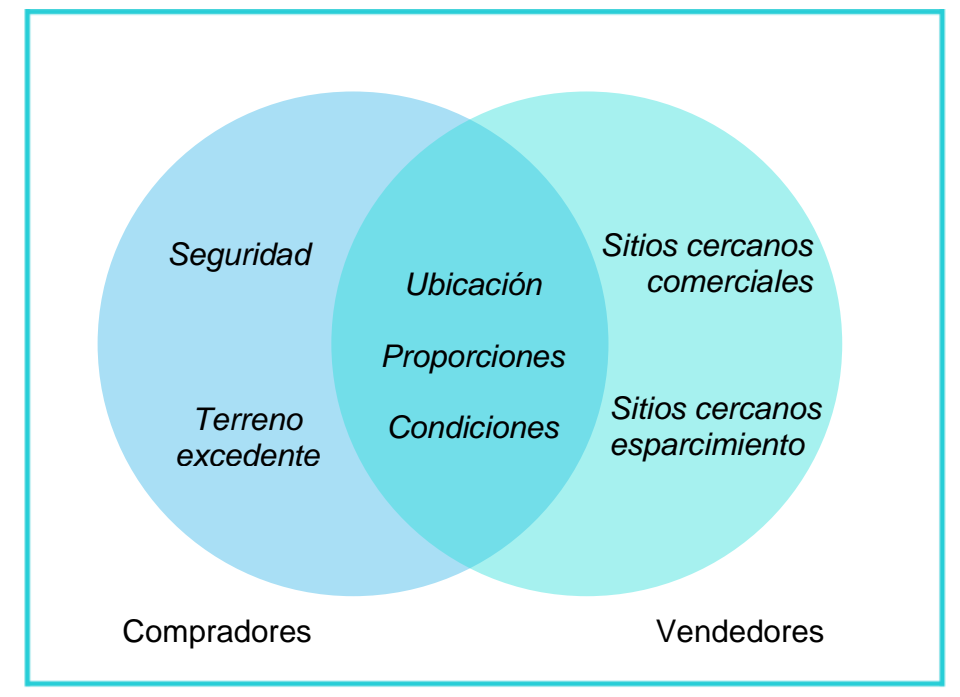

Fig. 5: Diagrama de Venn sobre la percepción de compradores y vendedores respecto a los factores que determinan el valor de una vivienda.

Por otro lado, los vendedores ni siquiera mencionaron esos factores, ellos hicieron énfasis en los sitios cercanos con los que cuentan las viviendas para determinar el precio de venta. En ese sentido, E4 (vendedor) justifica el precio de venta elevado de la siguiente manera: "Allá en dónde está la casa que vendí, el camión pasa por la casa, el seguro está cerca, la laguna, la escuela, el ITSON, las escuelas primarias o secundarias están ahí cerquitas (sic), tiene muchas cosas buenas que pueden influir en la vida de los compradores que vivan ahí, por el fácil acceso al transporte público y sitios cercanos que ya mencioné" (E4). Las opiniones entre compradores y vendedores difieren debido al tipo de experiencias y percepciones con los que cuentan. En ese sentido, la categoría sobre la percepción de los factores se ve relacionada con el contexto actual del individuo y sus experiencias. Los compradores consideran importante a la seguridad y al terreno excedente por sus intereses, es decir, buscan vivir en un sitio libre de delincuencia y en el que exista la posibilidad de expandir la construcción en un futuro, el siguiente fragmento de la entrevista a E1 (comprador) muestra su percepción sobre la importancia de la seguridad y el terreno excedente según sus intereses y experiencias: "Hubo un factor muy importante que no contemplé que fue la seguridad que había en la zona y al final terminé arrepintiéndome de dicha compra de la vivienda, por eso la regresé, por la poca seguridad que había ahí. ...El terreno excedente es uno de los factores más importantes al momento de determinar el valor o el costo de la vivienda... antes de comprarla se debe ver que sea lo suficientemente amplio como para expandirse o ampliarse de alguna manera la casa, es muy importante ver más allá, a largo plazo, por si crece la familia en algún momento tener terreno suficiente para poner uno o más cuartos" (E1). La percepción sobre las instalaciones (eléctricas, agua potable, saneamiento, servicios de telecomunicaciones) y los vecinos con los que cuenta la vivienda fueron bastante dispersas y dependen de otros subfactores más específicos dentro de cada tipo de participante, es por ello que no se incluyen en la Figura 5. Sin embargo, se explica la perspectiva de cada uno de esos factores como respuesta a las dos últimas preguntas de investigación.

\section{Factores de mayor y menor impacto por parte de los compradores}

Los compradores de la región sur del estado de Sonora que fueron entrevistados consideran como factores de mayor impacto a la ubicación, las proporciones, las condiciones, la seguridad y el terreno excedente. En la sección anterior se expusieron los motivos por los cuales dichos factores son catalogados como factores de mayor impacto o determinantes del valor de viviendas. Ahora bien, los factores considerados como factores de menor impacto son: Los sitios cercanos comerciales y de esparcimiento y las instalaciones de la vivienda (Ver Figura 6). E7 (comprador) comenta que no percibe relación entre los sitios cercanos y el costo de las viviendas: "Pues yo digo que con el costo no influyen, aunque ahora hace mucho auge eso de los sitios cercanos al fraccionamiento, ahora que hicieron la plaza comercial ahí cerca... No creo que influya en el costo pero en las personas sí, por ejemplo, dicen: 'está cerca de la Plaza Sendero ahí voy y compro'...” (E7). 
Las instalaciones de la vivienda son consideradas como un factor flexible que no influye realmente en el costo de la vivienda por parte de los compradores: “...No creo realmente que haya una relación entre las condiciones de las instalaciones y el costo de la misma. Es opcional al estimar el costo porque esas son cosas que se pueden reparar después... es algo que se puede reemplazar fácilmente. Claro que implica un costo pero es algo que se mira después de realizada la compra y se va arreglando poco a poco, conforme se tenga la posibilidad" (E1).

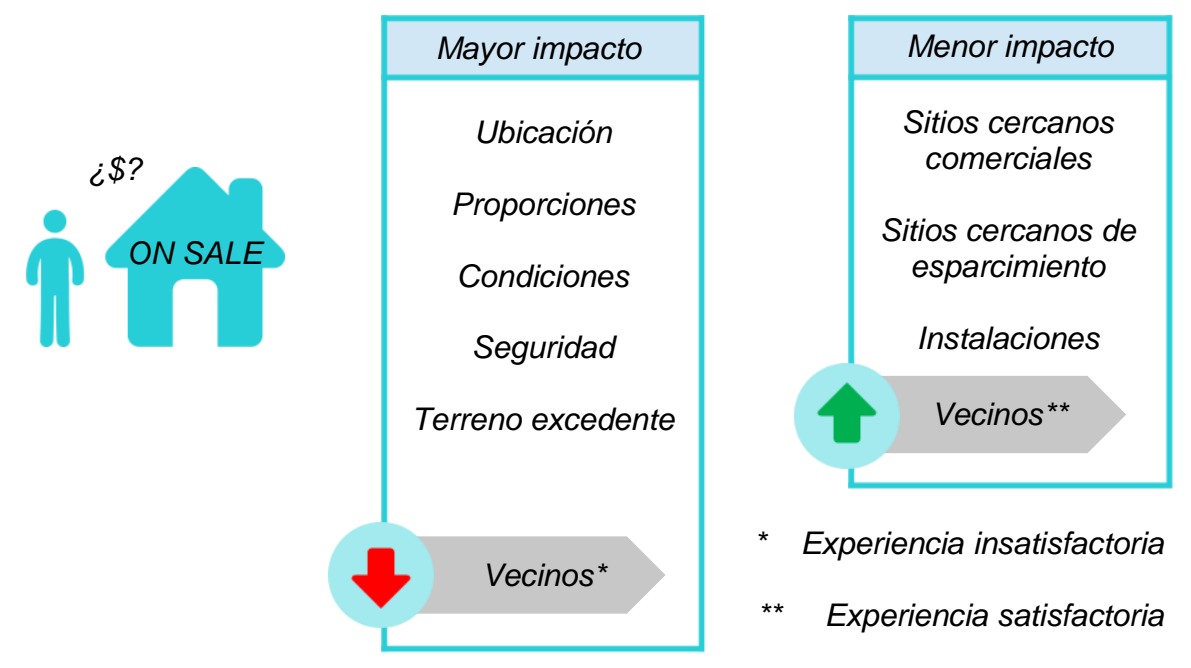

Fig. 6: Factores de mayor y menor impacto percibidos por compradores de viviendas.

La percepción y el nivel de impacto de los vecinos o el tipo de vecinos con los que cuenta una vivienda varía. Esto depende del tipo de experiencia previa con la que cuente el comprador en cuestión. Es decir, si el comprador ha tenido una experiencia insatisfactoria con los vecinos, entonces va a catalogar al "factor vecinos" como de mayor impacto para estimar el valor de alguna vivienda. E1 comenta que su experiencia con los vecinos ha sido insatisfactoria: "Mi experiencia no fue buena puesto que... me dejé llevar por la calle y no me fijé en los vecinos que tenía dicha vivienda o en el vecindario... tiempo después de que compré dicha casa...ya estaba pensando en mudarme ahí pero me di cuenta de que el barrio era realmente peligroso y los vecinos se veían peligrosos, me dio mucho miedo porque yo tengo dos hijas y preferí regresar esa casa y perdí la inversión que le había hecho con el piso y todo eso" (E1).

También menciona cómo eso ha influido en su percepción sobre la relación de los vecinos y el costo de las viviendas: "Hay un tipo de vecinos que son problemáticos... Por el riesgo que hay en el vecindario debería valer menos la casa, por eso te menciono que percibo esa relación entre esos dos puntos que son el tipo de vecinos y el costo de la casa" (E1). Por el contrario, si el comprador ha tenido una experiencia satisfactoria o neutral con los vecinos, entonces va a catalogar al "factor vecinos" como de menor impacto o como factor flexible para estimar el valor de alguna vivienda. E7 comenta que su experiencia con los vecinos ha sido satisfactoria: "Mi experiencia ha sido buena. Pues ahorita se ve que son vecinos tranquilos y que están al pendiente de las demás casas..." (E7). Debido a ello, no toma en cuenta o no cree que exista una relación entre los vecinos con los que cuenta una vivienda y el costo de la misma: "Pues no creo que los vecinos tengan algo que ver como tal con el costo de una vivienda... más bien segmentan por zonas de distintos niveles económicos en el fraccionamiento, haz de cuenta que por eso hay varias casas, hay unas más baratas y otras más caras, obviamente el tipo de persona no es la misma" (E7).

\section{Factores de mayor y menor impacto por parte de los vendedores}

Los vendedores de viviendas de la región sur del estado de Sonora que fueron entrevistados no mencionan la seguridad ni terreno excedente como posibles factores de mayor o de menor impacto, más bien se basan en los factores clave o estándar para determinar el valor de las viviendas. Consideran como factores de mayor impacto a la ubicación, las proporciones, las condiciones y los sitios cercanos con los que cuenta la vivienda. En la sección anterior se expusieron los motivos por los cuales dichos factores son catalogados como factores de mayor impacto o determinantes del valor de viviendas. Ahora bien, el factor considerado como factor de menor impacto fue el de vecinos de la vivienda (Ver Figura 7). E9 comenta que el tipo de vecinos no influye en el precio de venta del inmueble: "Yo creo que sería más importante sobre la zona como ya mencioné. Sobre los vecinos, no tanto. Porque si te vas a una zona de un nivel estable donde no haya mucho vandalismo ni delincuencia, creo yo que los vecinos importan muy poco... Ya es a criterio de cada quién, pero creo que no influye para estimar el precio de venta del inmueble." (E9). 


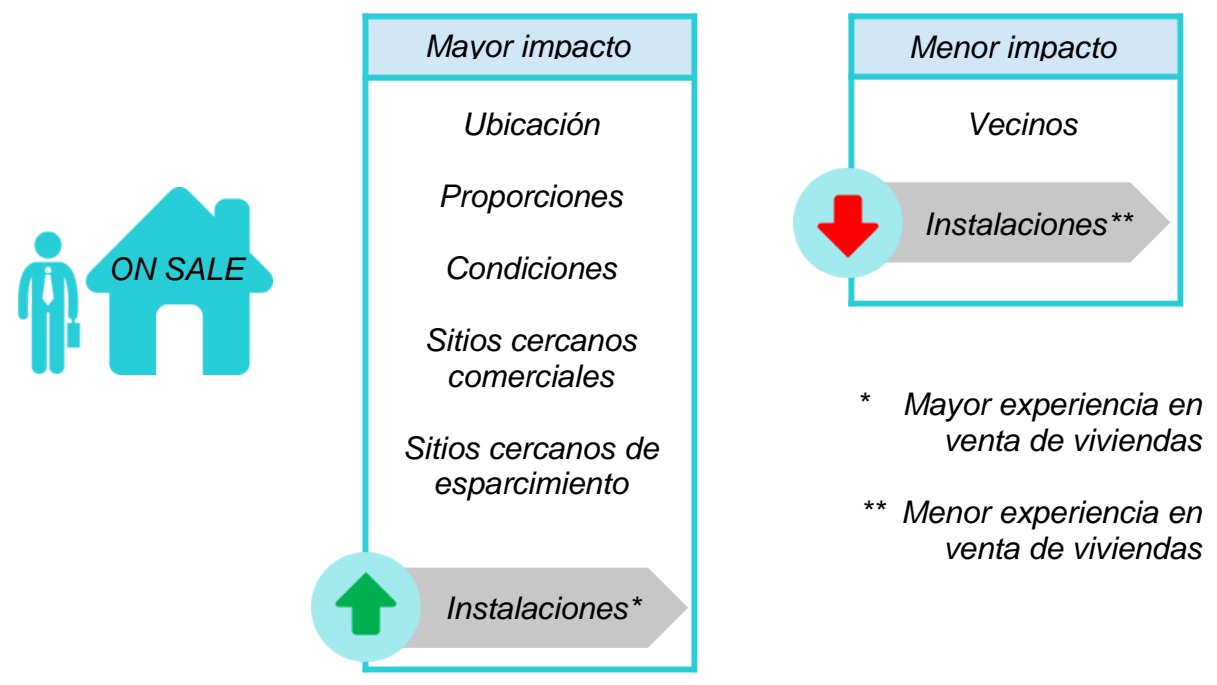

Fig. 7: Factores de mayor y menor impacto percibidos por vendedores de viviendas.

La percepción y el nivel de impacto en cuanto a las instalaciones (eléctricas, agua potable, saneamiento, servicios de telecomunicaciones) de la vivienda varía dependiendo del nivel de experiencia en la venta de viviendas del vendedor. Es decir, si el vendedor posee una mayor experiencia en cuanto a la venta de viviendas, entonces va a catalogar al "factor instalaciones" como de mayor impacto para estimar el valor de alguna vivienda. El participante E9 quién posee una mayor experiencia en cuanto a la compra de viviendas menciona lo siguiente: "Es muy importante... las instalaciones son algo que se tiene que considerar. También, si tiene algunos servicios, por ejemplo, unos amigos acaban de comprar una casa y, ni Telcel ni Megacable llega para allá.... en los mismos valores catastrales te viene todo eso, si cuenta con todos los servicios e instalaciones o cuenta con algunos servicios, si tienes qué hacer fosa o no...” (E9).

Por el contrario, si el vendedor posee una menor experiencia en cuanto a la venta de viviendas, entonces va a catalogar al "factor instalaciones" como de menor impacto para estimar el valor de alguna vivienda. E4 posee conocimientos básicos sobre el tema y comenta lo siguiente al respecto: "...Sería secundario eso de las instalaciones de las tuberías o eléctricas, no lo considero tan importante porque hay otras cosas más importantes para estimar el precio de venta de la casa... porque eso es punto y aparte, se puede arreglar o mejorar poco a poco por parte de los compradores... es de los factores flexibles que mencionaba en la pregunta anterior..." (E4).

\section{DISCUSIÓN}

Durante el proceso de la revisión de literatura, se encontraron numerosas investigaciones cualitativas en donde los autores emplean diversos métodos de análisis de datos cualitativos, tales como la teoría fundamentada o el análisis de contenidos, para conocer la percepción de las personas respecto a temas de interés en diversas áreas como la medicina (Figueira et al., 2009; Bedoya et al., 2017), psicología (Festen et al., 2014), tecnología (Riley et al., 2009; Bravo et al., 2018), gestión empresarial (Oliveira et al., 2016; Padilla et al., 2017; Pérez-Benedito et al., 2017), estudio de mercados (Kamagahara et al., 2016), entre otros. Sin embargo, se identificó cierta escasez respecto a este tipo de estudios cualitativos enfocados al contexto de bienes raíces. Kibet et al. (2016) realizó un estudio híbrido con rasgos cualitativos y cuantitativos para demostrar que los factores socioculturales influyen en la inversión inmobiliaria. Por otro lado, Kakulu (2014) propone una serie de estrategias para llevar a cabo un análisis cualitativo en el contexto inmobiliario. Si bien ambos trabajos involucran el tema de las investigaciones cualitativas y de bienes raíces, ninguno de ellos lleva a cabo un estudio para conocer la percepción de las personas en cuanto a la compra y venta de viviendas. En contraste, haciendo uso del método de la teoría fundamentada, en el presente trabajo se analizaron las percepciones de compradores y vendedores de viviendas para conocer cuáles factores son considerados de mayor y menor impacto durante el proceso de valuación de inmuebles.

El análisis obtenido de este estudio indica que, tanto compradores como vendedores de viviendas en la región sur del estado de Sonora consideran a la ubicación, las proporciones y las condiciones de las viviendas como factores fundamentales al momento de llevar a cabo la estimación del precio de alguna vivienda. Más específicamente, los compradores perciben a la ubicación, las proporciones de la vivienda, las condiciones de la vivienda, la seguridad en la zona y al terreno excedente como factores de mayor impacto; los sitios cercanos a la vivienda y el tipo de instalaciones con las que cuenta la vivienda son considerados factores de 
menor impacto. Por otro lado, los vendedores perciben a la ubicación, las proporciones, las condiciones y los sitios cercanos de la vivienda como factores de mayor impacto y consideran irrelevante o de menor impacto el tipo de vecinos. De igual modo, durante la fase de análisis de los datos se encontró que existen más dimensiones del fenómeno de estudio que pueden ser más profundamente analizadas en trabajos futuros.

Los resultados de este estudio beneficiarán a las personas de la región sur del estado de Sonora relacionadas con el contexto de bienes raíces; robusteciendo el método de valuación de viviendas al conocer la influencia percibida para cada uno de los factores involucrados en el proceso de estimación de precios. También proporcionará las bases necesarias para establecer las variables o parámetros a contemplar en un algoritmo predictivo para la estimación del precio de las viviendas en la región. Así mismo, este trabajo puede servir de guía para futuros estudios cualitativos relacionados con bienes raíces que deseen replicar el método utilizado y analizar datos obtenidos de muestras más grandes.

\section{CONCLUSIONES}

De acuerdo con los resultados obtenidos, se concluye lo siguiente: 1) Las preguntas de investigación planteadas al inicio de este trabajo de investigación fueron resueltas satisfactoriamente mediante la aplicación del método de la teoría fundamentada; 2) además, se encontró que los factores determinantes del valor de una vivienda desde la perspectiva de compradores y vendedores de viviendas son principalmente: la ubicación, las proporciones y las condiciones de la vivienda; y 3) el impacto del resto de los factores analizados varía dependiendo de distintas situaciones, tal como el perfil del participante (vendedor o comprador), el nivel de experiencia (mayor o menor) en cuanto a la venta y valuación de viviendas por parte de los vendedores e incluso el tipo de experiencia con los vecinos (satisfactorio e insatisfactorio) por parte de los compradores de inmuebles.

\section{REFERENCIAS}

Agee, J., Developing Qualitative Research Questions, A Reflective Process, doi: 10.1080/09518390902736512, International Journal of Qualitative Studies in Education, 22(4), 431-447 (2009)

Arias, C., Enfoques Teóricos sobre la Percepción que Tienen las Personas, ISSN: 2500-705X, Horizontes Pedagógicos, 8(1), 9-22 (2006)

Ayuntamiento de Cajeme, Programa Parcial de Crecimiento Urbano Zona Sureste y Corredor Aeropuerto (2011)

Barnes, D.M., An Analysis of the Grounded Theory Method and the Concept of Culture, doi: 10.1177/104973239600600309, Qualitative Health Research, 6(3), 429-441 (1996)

Basit, T., Manual or electronic? The role of coding in qualitative data analysis, doi: 10.1080/0013188032000133548, Educational Research, 45(2), 143-154 (2003)

Basu, S. y T. G. Thibodeau, Analysis of Spatial Autocorrelation in House Prices, doi: 10.1023/a:1007703229507, The Journal of Real Estate Finance and Economics, 17(1), 61-85 (1998)

Bedoya, E. A., D. D. Sierra, C. A. Severiche y M. D. J. Meza, Diagnóstico de Bioseguridad en el Sector Sanitario del Departamento de Bolívar, Norte de Colombia, doi: 10.4067/S0718-07642017000500021, Información Tecnológica, 28(5), 225-232 (2017)

Bell, R. y M. P. Bell, Real Estate Research Methods, Appraisal Journal, 83(4), 310-318 (2015)

Bourassa, S.C., E. Cantoni y M. Hoesli, Spatial Dependence, Housing Submarkets, and House Price Prediction, doi: 10.1007/s11146-007-9036-8, The Journal of Real Estate Finance and Economics, 35(2), 143-160 (2007)

Bravo, C. J., P. E. Ramírez y J. Arenas, Aceptación del Reconocimiento Facial Como Medida de Vigilancia y Seguridad: Un Estudio Empírico en Chile, doi: 10.4067/S0718-07642018000200115, Información Tecnológica, 29(2), 115-122 (2018)

CANADEVI, Cámara Nacional de la Industria de Desarrollo y Promoción de Vivienda, Estadísticas y Publicaciones: Cajeme, (2015)

Carterette, E.C. y M.P. Friedman, Manual de Percepción: Raíces Históricas y Filosóficas, ISBN: 96824114839789682411489, (1982)

Clifford, N., M. Cope, T. Gillespie y S. French, Key Methods in Geography, ISBN: 978-1-4462-9858-9, Sage (2016)

Cohen, D. y B. Crabtree, Qualitative Research Guidelines Project, Robert Wood Johnson Foundation (2006)

Daniels, H. y B. Kamp, Application of MLP Networks to Bond Rating and House Pricing, doi: 10.1007/s005210050025, Neural Computing \& Applications, 8(3), 226-234 (1999)

Del Giudice, V., P. De Paola y F. Forte, Using Genetic Algorithms for Real Estate Appraisals, doi: 10.3390/buildings7020031, Buildings, 7(2), 31-40 (2017)

Esquer, J.A., Estimación del Precio de Venta de la Vivienda en la Ciudad de Hermosillo Aplicando el Método de Precios Hedónicos, (U. d. Sonora, Editor), Tesis de grado, Posgrado en Ciencias de la Ingeniería, Ingeniería Civil, UNISON (2012) 
Festen, H., K. Schipper y otros cuatro autores, Parents' Perceptions on Offspring Risk and Prevention of Anxiety and Depression: A Qualitative Study, doi: 10.1186/2050-7283-2-17, BMC Psychology, 2(1), 1-17 (2014)

Figueira, T.R., E. F. Ferreira, V. T. Schall y C. M. Modena, Women's Perceptions and Practices Regarding Prevention and Health Promotion in Primary Healthcare, doi: 10.1590/s0034-89102009005000081, Revista de Saude Publica, 43(6), 937943 (2009)

Glaser, B. y A. Strauss, Discovery of Grounded Theory: Strategies for Qualitative Research, ISBN: 9781351522168 , Routledge (2017)

Grams, G. D., Rudiments in the Use of 'Grounded Theory': A Working Guide, Unpublished Manuscript, University of British Columbia (2001)

Haurin, D.R. y D. Brasington, School Quality and Real House Prices: Inter-and Intrametropolitan Effects, doi: 10.1006/jhec.1996.0018, Journal of Housing economics, 5(4), 351-368 (1996)

IMIP, Instituto Municipal de Investigación y Planeación Urbana de Cajeme, Portal de transparencia, Antecedentes (2017)

INEGI, Instituto Nacional de Estadística y Geografía, Censo de población y vivienda 2010, Banco de indicadores, Sonora, Cajeme (2010)

INEGI, Instituto Nacional de Estadística y Geografía, Censo de población y vivienda 2015, Banco de indicadores, Sonora, Cajeme (2015)

INEGI, Instituto Nacional de Estadística y Geografía, Censo Nacional de Gobiernos Municipales y Delegacionales, Seguridad Pública y Justicia, Sonora, Cajeme (2012)

Kakulu, I. I., Qualitative Research Strategies and Data Analysis Methods in Real Estate Research - An innovative approach using the BB Model, Estate Management Department Workshop, At Federal Polytechnic, Nekede, Owerri (2014)

Kamagahara, Y., T. Takeda y otros cinco autores, Qualitative Analysis of the Customer Satisfaction at the Dental Clinics, doi.org/10.1007/978-3-319-40247-5_24, In International Conference on Digital Human Modeling and Applications in Health, Safety, Ergonomics and Risk Management, Springer, Cham, 229-242 (2016)

Khandkar, S.H., Open coding, University of Calgary, 23-31 (2009)

Kibet, F., E. Nambuswa y G. S. Namusonge, Effect of Social-Cultural Factors on Real Estate Investment: A Survey of Kisumu City, European Journal of Business and Management, 8(29), 85-91 (2016)

Martínez-Salgado, C., El muestreo en investigación cualitativa: principios básicos y algunas controversias, doi: 10.1590/S1413-81232012000300006, Ciência \& Saúde Coletiva, 17, 613-619 (2012)

Muylaert, C. J., V. Sarubbi y otros tres autores, Narrative Interviews: An Important Resource in Qualitative Research, doi: 10.1590/s0080-623420140000800027, Revista da Escola de Enfermagem da USP, 48(SPE2), 184-189 (2014)

Oliveira, E., T. Conte, M. Cristo y E. Mendes, Software Project Managers' Perceptions of Productivity Factors: Findings from a Qualitative Study, doi:10.1145/2961111.2962626, In Proceedings of the 10th ACM/IEEE International Symposium on Empirical Software Engineering and Measurement, ACM (2016)

Padilla, C.P., D. X. Arévalo, M.A. Bustamante y C.L. Vidal, Responsabilidad Social Empresarial y Desempeño Financiero en la Industria del Plástico en Ecuador, doi: 10.4067/S0718-07642017000400012, Información Tecnológica, 28(4), 93-102 (2017)

Pérez-Benedito, M.A., L. Porcuna-Enguix y R. Porcuna-Enguix, Los Mapas Contables de Gestión de las empresas cotizadas chilenas: Análisis Cualitativo, doi: 10.4067/S0718-07642017000100016, Información Tecnológica, 28(1), 161170 (2017)

Riley, C., K. Buckner, G. Johnson y D. Benyon, Culture \& Biometrics: Regional Differences in the Perception of Biometric Authentication Technologies, doi: 10.1007/s00146-009-0218-1, Al \& society, 24(3), 295-306 (2009)

Schram, J.F., Real Estate Appraisal, ISBN: 1887051252-9781887051255, Rockwell Publishing (2006)

Strauss, A. y J. Corbin, Bases de la Investigación Cualitativa: Técnicas y Procedimientos para Desarrollar la Teoría Fundamentada, Medellín, Universidad de Antioquia, (2002)

Strauss, A. y J. Corbin, Basics of Qualitative Research: Procedures and Techniques for Developing Grounded Theory, Sage (1998)

Strauss, A. y J. Corbin, Grounded Theory Methodology, Handbook of Qualitative Research, 17, $273-285$ (1994)

Wells, K., The strategy of Grounded Theory: Possibilities and Problems, doi:10.1093/swr/19.1.33, Social Work Research 19(1), 33-37 (1995)

Zietz, J., E.N. Zietz y G. S. Sirmans, Determinants of House Prices: A Quantile Regression Approach, doi: 10.1007/s11146007-9053-7, The Journal of Real Estate Finance and Economics, 37(4), 317-333 (2008) 